OPEN

SUBJECT AREAS:

METHODS

NEUROSCIENCE

STATISTICAL PHYSICS,

THERMODYNAMICS

AND NONLINEAR

DYNAMICS

MATHEMATICS

Received

1 February 2012

Accepted

8 May 2012

Published

25 May 2012

Correspondence and requests for materials should be addressed to K.I. (koji@sat.t.u-tokyo. ac.jp)

* These authors equally contributed to this

work.

\section{Characterizing global evolutions of complex systems via intermediate network representations}

\author{
Koji Iwayama ${ }^{1,2 *}$, Yoshito Hirata ${ }^{2 *}$, Kohske Takahashi ${ }^{3}$, Katsumi Watanabe $^{3}$, Kazuyuki Aihara $^{2}$ \\ \& Hideyuki Suzuki
}

${ }^{1}$ FIRST, Aihara Innovative Mathematical Modelling Project, JST, 4-6-1 Komaba, Meguro-ku, Tokyo, Japan, ${ }^{2}$ Institute of Industrial Science, The University of Tokyo, 4-6-1 Komaba, Meguro-ku, Tokyo, Japan, ${ }^{3}$ Research Center for Advanced Science and Technology, The University of Tokyo, 4-6-1 Komaba, Meguro-ku, Tokyo, Japan.

Recent developments in measurement techniques have enabled us to observe the time series of many components simultaneously. Thus, it is important to understand not only the dynamics of individual time series but also their interactions. Although there are many methods for analysing the interaction between two or more time series, there are very few methods that describe global changes of the interactions over time. Here, we propose an approach to visualise time evolution for the global changes of the interactions in complex systems. This approach consists of two steps. In the first step, we construct a meta-time series of networks. In the second step, we analyse and visualise this meta-time series by using distance and recurrence plots. Our two-step approach involving intermediate network representations elucidates the half-a-day periodicity of foreign exchange markets and a singular functional network in the brain related to perceptual alternations.

he developments of measurement techniques have resulted in the generation of very high-dimensional time series for complex systems. Such examples include measurements obtained from economic trades of various currencies and multichannel signals of neural activities measured by magnetoencephalography. Interactions among elements, each of which is represented by a time series, affect the global behaviour of such systems ${ }^{1-3}$. Although many methods for analysing interactions between two or more time series have been proposed ${ }^{4-9}$, there are only a few methods that provide an intuitive idea on the underlying dynamics of the entire system. These studies have focussed on stationary and time-independent interactions. It should be noted that interactions in real complex systems are generally nonstationary and evolve over time more slowly than the time evolution of their elements.

Here, we propose a method for characterizing the global dynamical aspects of high-dimensional systems using recurrence plots ${ }^{10,11}$, which visualise time series data on a two-dimensional plane. In the recurrence plots, both the two axes represent time. If and only if two states, at time $i$ and $j$, are sufficiently close, we plot a point at $(i, j)$ and $(j, i)$. Trivially, points are plotted at $(i, i)$ for all $i$. Recurrence plots provide relevant information on a time series, including its determinism and periodicity. We use two types of recurrence plots-short-term local recurrence plots and long-term global recurrence plots. First, we use short-term local recurrence plots to examine bivariate interactions ${ }^{9}$ of all pairs of time series for short-term periods to produce a meta-time series of networks as an intermediate representation of the slow global dynamics for an entire system (see Figs. 1a and 1b). Second, we use a long-term global recurrence plot to analyse the metatime series of networks by introducing the distance between the networks (see Fig. 1c). In this study, we used the hamming distance ${ }^{12}$, which is defined as the total number of pairs of nodes where two networks are different. Defining a distance between networks enables us to apply various methods to time-evolving networks, for instance, the estimation of the maximal Lyapunov exponent ${ }^{13}$, mapping of networks to multidimensional space ${ }^{14}$, and visualisation using recurrence plots.

\section{Results}

In this section, we present the results of the application of the proposed method for two numerical simulations and two real data. For details of the analysis, see Methods. 


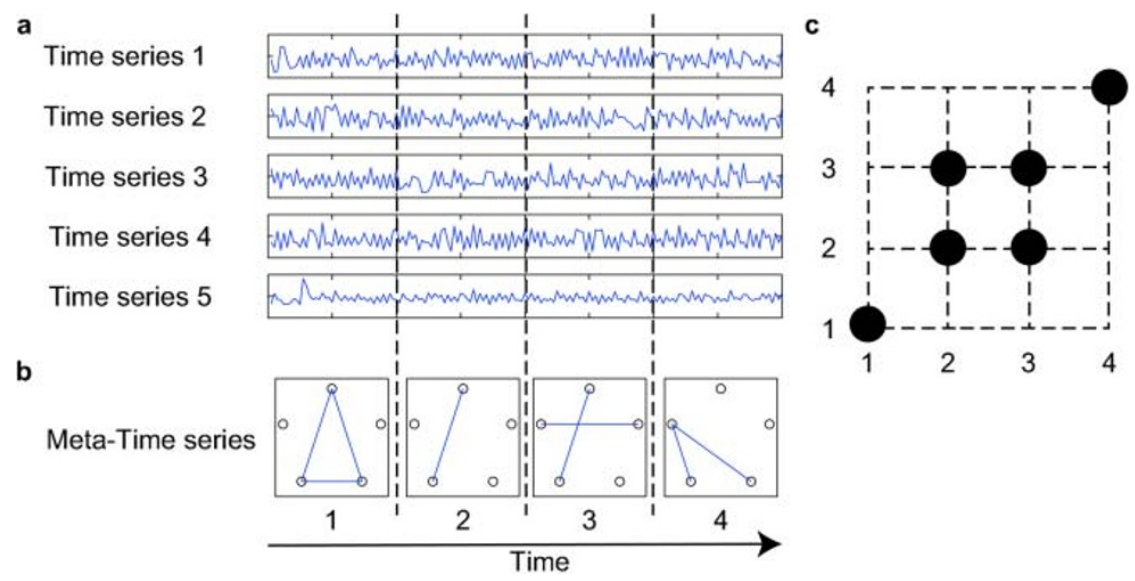

Figure $1 \mid$ Characterizing global dynamical aspects through network constructions. (a) We assume that we can observe high-dimensional time series data from a complex system. (b) Second, we obtain a meta-time series of networks by using correlation of short-term recurrence plots. Each panel is the estimated network, where each node corresponds to one time series during the corresponding time-segment. (c) Third, we obtain the long-term global recurrence plot of the meta-time series shown in (b). Because networks at time 2 and 3 are similar in the sense that the hamming distance is equal to 1 , points are plotted at $(2,3)$ and $(3,2)$ in the long-term global recurrence plot of $(\mathrm{c})$. We can characterize various kinds of information pertaining to the global dynamics of the measured complex system from this long-term global recurrence plot.

Numerical simulations. We simulated coupled logistic maps on two types of artificial time-evolving networks.

First, we considered the network consisting of five nodes that evolves periodically. The period of time evolution is 8000 steps. We applied the proposed method to a time series $\boldsymbol{x}(t)$ and obtained the long-term global recurrence plot of the meta-time series of the time-evolving network. From this recurrence plot, we calculated the $\tau$-recurrence rate ${ }^{15}$ defined in Eq. (5) in Methods, which characterizes the periodicity of the meta-time series. The plot of averages and standard deviations of the $\tau$-recurrence rate of 100 simulations showed peaks at approximately 8000 and 16000 (see Supplementary Fig. S1 online). The peak values of $\tau$-recurrence rates are significantly large. This indicates the periodicity of the global dynamics of the system, and the period of the global dynamics is about 8000 steps, which is consistent with the time evolution of the network.

Second, we prepared five instances of topologies of randomly connected networks consisting of 10 nodes where each pair of nodes is connected with the probability 0.4 . The time-evolving network begins with one of these instances and randomly switches to another instance. To validate the effectiveness of the proposed method, we compared the precision of the long-term global recurrence plot obtained by the proposed method with that of the recurrence plot obtained from the raw time series $\mathbf{x}(t)$. Average values and standard deviations of the precision of long-term global and raw recurrence plots are $0.69 \pm 0.1$ and $0.29 \pm 0.07$, respectively (see Supplementary section S1.2). The precision of long-term global recurrence plots is significantly higher than that of raw recurrence plots $(p=3.9 \times$ $10^{-18}$, Wilcoxon signed-rank test). This result suggests that intermediate network representations of time series contain information pertaining to the global dynamics, which could not be directly extracted from raw time series.

Foreign exchange. We analysed the tick data of foreign exchanges obtained from the EBS Service Company Limited, with a timeresolution of $1 \mathrm{~s}$. In our study, we considered the trades of Japanese yen (JPY), Great Britain pound (GBP), Euro (EUR), Canadian dollars (CAD), Swiss francs (CHF), Australian dollars (AUD), New Zealand dollars (NZD), Hong Kong dollars (HKD), Singapore dollars (SGD), Mexican pesos (MXN), gold (XAU), and silver (XAG) with US dollars. The dataset used here is available from the company above.

The meta-time series of networks of different currencies is illustrated in Fig. 2a, where each box shows a network for a particular time duration. In Fig. 2b, we show the long-term global recurrence plot of the time-evolving network mentioned above. From this recurrence plot, we calculated the $\tau$-recurrence rate ${ }^{15}$ as with the first numerical simulation. The plot of the $\tau$-recurrence rate against $\tau$ is shown in Fig. 2c. Half-a-day peaks in this plot indicate the half-a-day periodicity.

Magnetoencephalography. For the second example of real data, we used magnetoencephalography (MEG). Seven subjects participated in this study. The procedures were in accordance with the Declaration of Helsinki. All participants signed the informed consent form. The MEG data used here were based on the data collected with approved procedures by the ethics committee of Kanazawa University. The stimulus comprised two images. In one image, two points were present on the upper right and lower left corners, and in the other image, two points were present on the upper left and lower right corners (motion quartet ${ }^{16}$; see Fig. 3a). This stimulus is ambiguous in that it induces the perception of either horizontal or vertical motion. The perception spontaneously and successively alternates between the two perceptions ${ }^{17}$. The motion-quartet ambiguous display was shown for $60 \mathrm{~s}$. The subjects were instructed to look at the centre of the stimulus and press a button with their right hand when their perception alternated. From the subjects lying on a bed, we recorded signals by MEG (PQ1160C; 160 channels; sampling rate is $1000 \mathrm{~Hz}$ ). The obtained signals were then band-pass filtered between 0 and $500 \mathrm{~Hz}$.

We constructed time-evolving networks of MEG data (see Fig. 3b). The long-term global recurrence plot of the time-evolving network of one subject is illustrated in Fig. 3c. It is clearly shown that only a few points were plotted just before a subject pressed the button (see the red vertical and horizontal lines in Fig. 3c). We also obtained similar results for all the other subjects; the recurrence plots showed sparse columns and rows just before or after the button was pressed. We define sparse rows (or columns) of a recurrence plot as rows (or columns) that have, at most, only one point. A sparse row indicates that a state similar to the state at the time corresponding to the sparse row does not appear in the time series. We call rows other than sparse rows as dense rows.

Table 1 compares the number of sparse rows around perceptual alternations with that while no perceptual alternation was reported. Specifically, 5 seconds before and after button presses were regarded as around perceptual alternations. The number of sparse rows around perceptual alternations is significantly large $(p=0.0083<$ 0.01 , chi-square test). 
a

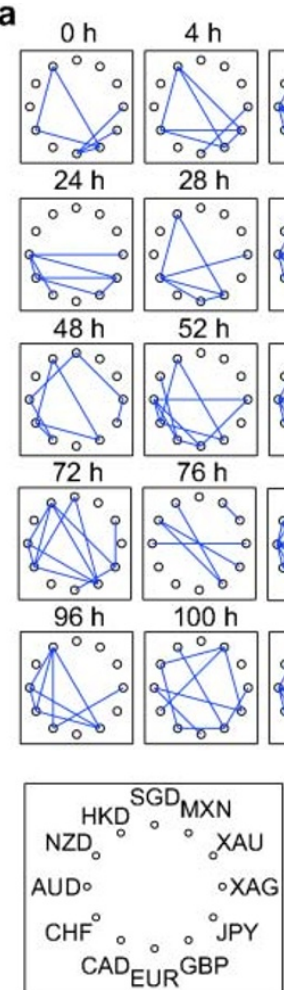

b

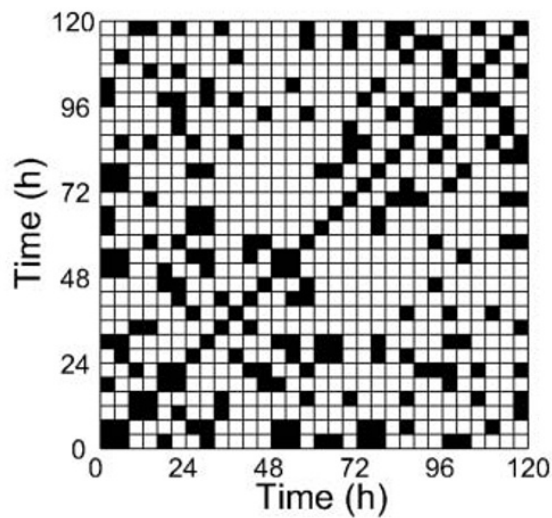

C

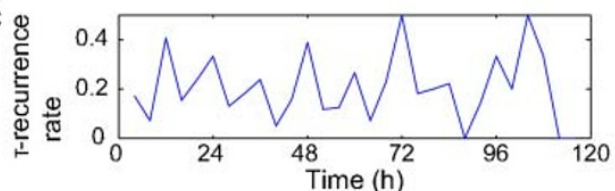

d

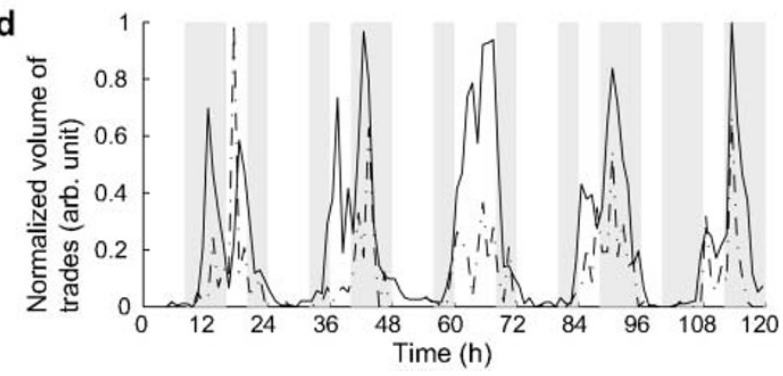

Figure $2 \mid$ Analysis of foreign exchange data. (a) The meta-time series of networks between different currencies from 9 July 2007 for five days. The arrangement of currencies is illustrated in the block on the bottom. In each block, the circles correspond to different currencies, and the blue lines represent the links between two currencies. (b) Long-term global recurrence plot of the time-evolving network shown in (a). (c) $\tau$-recurrence rate of the long-term global recurrence plot shown in (b). (d) The volume of trades of Great Britain pound GBP (the solid line) and silver XAG (the dash-dotted line) with US dollars. The shadowed areas indicate the times when the link exists between GBP and XAG.

a

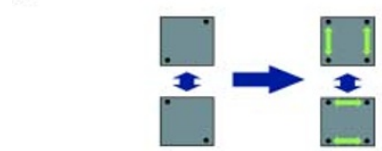

c

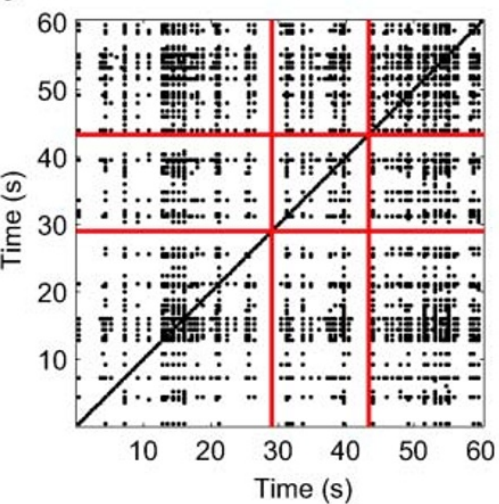

b

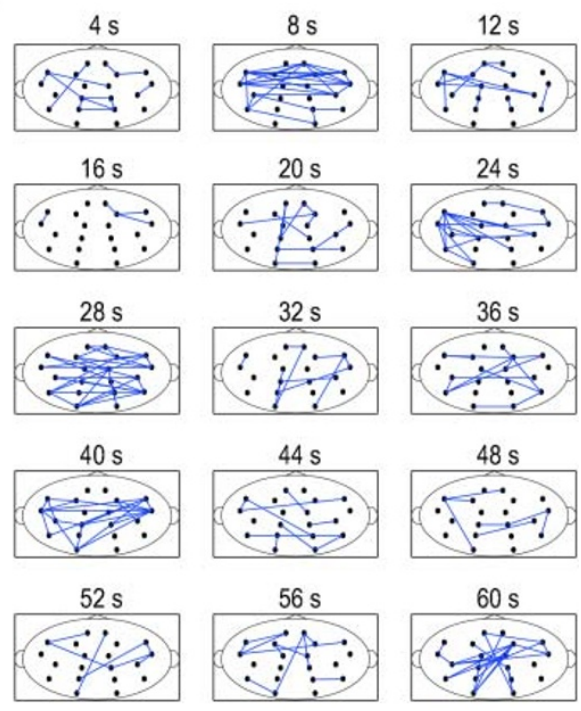

Figure 3 Analysis of magnetoencephalography data. (a) The ambiguous apparent motion used for the experiment. We showed the two figures on the left alternately. Subjects perceived this figure as either horizontal or vertical motion of two points. (b) The meta-time series of networks consisting of different areas in the brain. 20 channels out of 160 channels are shown. (c) Long-term global recurrence plot of the time-evolving network shown in (b). The red vertical and horizontal lines indicate the time at which a subject pressed the button to inform the change of perceived motion. Clearly, fewer points are plotted just before the red lines. 


\begin{tabular}{|c|c|c|}
\hline & sparse rows & dense rows \\
\hline $\begin{array}{l}\text { around perceptual alternations } \\
\text { no perceptual alternation }\end{array}$ & $\begin{array}{l}179(48 \%) \\
269(40 \%)\end{array}$ & $\begin{array}{l}192(52 \%) \\
410(60 \%)\end{array}$ \\
\hline
\end{tabular}

\section{Discussion}

Recent developments in measurement techniques enabled us to observe a large number of time series simultaneously. To globally characterize such high-dimensional time series, we proposed a method for analysing the meta-time series of interactions among individual time series. Using the proposed method, first, we construct time-evolving networks by estimating the interactions among time series using short-term recurrence plots. Second, we introduce the distance for networks, and using this distance, we visualise the dynamics of time-evolving networks with long-term global recurrence plots.

Numerical simulations showed the validity of the proposed method. The proposed method revealed the underlying dynamics of time-evolving networks. It is extremely difficult to estimate the network structure from time series. In fact, the estimated network structure using short-term local recurrence plots was not necessarily exact. Nevertheless, long-term global recurrence plots reflected actual time evolution of networks. The second case of numerical simulations showed that the meta-time series contains the information pertaining to the global dynamics that is difficult to observe through the raw time series by themselves. In the raw time series, the global dynamics is enveloped by individual dynamics on each node. Discarding information pertaining to individual dynamics by constructing meta-time series allows us to extract information pertaining to the global dynamics, which characterized the entire system.

The proposed method revealed that the network among currencies in the foreign exchange markets exhibits half-a-day periodicity. On the other hand, the volume of trades of each currency does not show half-a-day periodicity; it shows daily periodicity. For example, Fig. $2 \mathrm{~d}$ shows the time series of the volume of trades of GBP and XAG, where the link shows the clearest half-a-day periodicity, with US dollars. Links between currencies tend to appear at the beginning and end of daily periodic peaks of the volume of trades. This implies that trades of currencies tend to correlate at the beginning and end of peaks of trades. Therefore, the link and the entire network exhibit half-a-day periodicity. The application of the conventional method to the entire foreign exchange market did not result in half-a-day periodicity (see Supplementary section S2.1).

The long-term global recurrence plots of the MEG data indicated that sparse rows are more likely to be associated with perceptual alternations. This indicates that singular networks that do not reappear in the meta-time series appear more frequently around perceptual alternations. It is known that synchronization among distributed areas in the brain plays an important role in the integration of the information processed in these areas and the perceptions ${ }^{18-20}$. Moreover, some patterns of transient synchronization were observed prior to perceptual alternation $\mathrm{s}^{21}$. Singular networks detected by the proposed method might reflect transient synchronization among channels associated with perceptual alternations. The distributions of numbers of motifs ${ }^{22,23}$ appearing in the meta-time series of networks, on the other hand, did not show any significant tendency associated with perceptual alternations in the data (see Supplementary section S2.2). Therefore, the variation in the topology of the network, rather than the motif distribution of the network, might be related to perceptual alternations.

Although we used the hamming distance for the analyses of real data, other distances can also be used. In fact, some other distances for the networks are proposed ${ }^{24,25}$. However, the calculations of these distances require high computational costs. Therefore, in our studies, we used the hamming distance.

The proposed method will provide deep insights into the dynamics of high-dimensional and complex systems, which are ubiquitous in the real world.

\section{Methods}

We proposed the method to characterize global evolutions of complex systems. We consider the case where we measure a large number of time series simultaneously from the complex system. The proposed method consists of two steps. The first step is the division of time series into shorter segments and the construction of a meta-time series of networks, and the second step is the characterization of this meta-time series. In the following section, we assume that the number of simultaneously measured time series is $N$ and the length of the time series is $T$. The number of segments into which the time series are divided is $M$. We denote the $k$ th time series by $\boldsymbol{x}^{k}(t)$ and the $m$ th segment of the $k$ th time series by $\boldsymbol{x}^{k m}(t)$. Moreover, we denote the distance between two states $\boldsymbol{x}$ and $\boldsymbol{y}$ by $d(\boldsymbol{x}, \boldsymbol{y})$. The meta-time series of networks constructed in the first step of the proposed method is denoted by $\boldsymbol{A}(m)$, where $A_{k l}(m)=1$ and $A_{k l}(m)=0$ indicate the existence and nonexistence of the link between the $k$ th and $l$ th time series at the $m$ th segment, respectively.

Recurrence plots. In the proposed method, we use two types of recurrence plots ${ }^{10,11}$, namely, short-term local recurrence plots used for the construction of a meta-time series of networks and long-term global recurrence plots used for analysing the metatime series of networks. Recurrence plots are effective tools for visualising time series on a two-dimensional plane.

The recurrence plot $R(x(t))$ of the time series $x(t)$ with a certain threshold $r$ is defined by the following binary matrix:

$$
R_{i, j}(\mathrm{x}(t))= \begin{cases}1, & \text { if } d(x(i), x(j))<r \\ 0, & \text { otherwise }\end{cases}
$$

In a figure, we plot a black dot at $(i, j)$ if $R_{i, j}=1$. Otherwise, we plot nothing at $(i, j)$.

Construction of a meta-time series of networks. Before analysing the global dynamics of the system, we estimate the time-dependent correlation between each pair of time series and construct meta-time series of networks. To construct the metatime series, we divide each time series into $M$ segments with length $S=T / M$. Subsequently, we construct the network where the node $k$ corresponds to the $k$ th time series for each segment. Then, we obtain the meta-time series $A(m)(m=1, \ldots, M)$. If the $m$ th segments of the $k$ th and $l$ th time series are significantly correlated, $A_{k l}(m)=1$. Otherwise, $A_{k l}(m)=0$

To test whether two time series are significantly correlated or not, we used shortterm local recurrence plots and their joint recurrence plots ${ }^{9,15,26}$. The joint recurrence plot $\boldsymbol{J}\left(\boldsymbol{x}^{k m}(t), \boldsymbol{x}^{l m}(t)\right)$ of the $m$ th segments of the $k$ th and $l$ th time series is defined as follows:

$$
J R_{i, j}\left(\boldsymbol{x}^{k m}(t), \boldsymbol{x}^{l m}(t)\right)=R_{i, j}\left(\boldsymbol{x}^{k m}(t)\right) \cdot R_{i, j}\left(\boldsymbol{x}^{l m}(t)\right) .
$$

Here, $R_{i, j}\left(\boldsymbol{x}^{k m}(t)\right)$ and $R_{i, j}\left(\boldsymbol{x}^{l m}(t)\right)$ are short-term local recurrence plots. If and only if black dots are plotted at $(i, j)$ in both recurrence plots, a black dot is plotted at $(i, j)$ of the joint recurrence plot. The stronger the correlation between two time series is, the more black dots are plotted in the joint recurrence plot of these time series. If two time series are independent, their recurrence plots are also independent. Hence, the number of points plotted in the strictly upper (or lower) triangular part of the joint recurrence plot $\boldsymbol{J}\left(\boldsymbol{x}^{\mathrm{km}}(t), \boldsymbol{x}^{l m}(t)\right)$ follows a binomial distribution of size $n=S(S-1) / 2$ and probability $q_{k m} q_{l m}$, where $q_{k m}$ and $q_{l m}$ are the rates of the points in the triangular parts of $\boldsymbol{R}\left(\boldsymbol{x}^{\mathrm{km}}(t)\right)$ and $\boldsymbol{R}\left(\boldsymbol{x}^{l m}(t)\right)$, respectively. If $n$ is sufficiently large,

$$
Z_{k l}(m)=\frac{\sum_{i>j} J R_{i, j}\left(x^{k m}(t), x^{l m}(t)\right)-n q_{k m} q_{l m}}{\sqrt{n q_{k m} q_{l m}\left(1-q_{k m} q_{l m}\right)}}
$$

approximately follows the normal distribution of mean 0 and variance 1 . When the $Z_{k l}(m)$ is sufficiently large ( 0.05 significance level), the $m$ th segments of the $k$ th and $l$ th time series are defined to be significantly correlated, and we set $A_{k l}(m)=1$.

Characterization of a meta-time series. To characterize the global dynamics of the system, we use a long-term global recurrence plot, which is a recurrence plot of a meta-time series $A(m)$. The definition of recurrence plots in Eq. (1) contains a distance between states at two times. Therefore, we need to introduce a distance between the networks of the meta-time series. We use the hamming distance ${ }^{12}$. The hamming distance between two networks $A(i)$ and $A(j)$ is defined as follows:

$$
d(\boldsymbol{A}(i), \boldsymbol{A}(j))=\sum_{k>l}\left\|A_{k l}(i)-A_{k l}(j)\right\| .
$$

This distance corresponds to the total number of pairs of nodes where two networks are different. Using this distance, we can obtain the long-term global recurrence plot of the meta-time series and characterize the global dynamics of the observed highdimensional complex system. 
We can obtain various types of information from recurrence plots. For example, in this study, we used $\tau$-recurrence rates ${ }^{15}$, which represent the $\tau$-periodicity of a time series. The $\tau$-recurrence rate of the recurrence plot of size $H$ is defined as

$$
R R_{\tau}=\frac{1}{H-\tau} \sum_{i=1}^{H-\tau} R_{i, i+\tau} .
$$

Application to numerical simulations. For numerical simulations, we used time series of states $s_{i}(t)$ in $d$-dimensional delay coordinates ${ }^{28}$ defined as

$$
\boldsymbol{s}_{i}(t)=\left(x_{i}(t), x_{i}(t+1), \ldots, x_{i}(t+d-1)\right)^{T} .
$$

We constructed delay coordinates without overlapping, i.e., $t=d, 2 d, \ldots$ We used the number of the nodes in the network for the dimension of delay coordinates. That is, $d=5$ for the first case, and $d=10$ for the second case. These time series in delay coordinates were divided into segments whose length is 200 . Then, the $m$ th segment of the node $k$ is defined as

$$
\begin{aligned}
\boldsymbol{s}^{k m}(t) & =\left(x_{k}(200 d(m-1)+t-d+1), \ldots, x_{k}(200 d(m-1)+t)\right)^{T} \\
\text { for } t & =d, 2 d, \ldots, 200 d .
\end{aligned}
$$

We set the threshold of short-term recurrence plots to obtain a probability of 0.05 for plotting a dot. If the number of black dots contained in the joint recurrence plot $J R\left(s^{k m}(t), s^{l m}(t)\right)$ is significantly large with the significance level $\alpha=0.01$, two nodes $k$ and $l$ were connected at time $m$, i.e., $A_{k l}(m)=1$.

In the first case, we selected the threshold of long-term global recurrence plots to obtain a probability of 0.1 or plotting a black dot. In the second case, the threshold of recurrence plots of meta-time series and raw time series are determined so that the total numbers of black dots in these recurrence plots become equal to the total number of black dots in the true recurrence plots of network patterns (see Supplementary section S1.2).

Application to real data. When we analysed the tick data of foreign exchanges, we divided the time series of each currency into segments with a length of $4 \mathrm{~h}$. To obtain short-term local recurrence plots, we further divided each segment into shorter windows whose length is $10 \mathrm{~min}$ and calculated the distances for the marked point process data ${ }^{27}$ between the pairs of shorter windows. Therefore, the size of each shortterm local recurrence plot is 24 . Then, we obtain the meta-time series $\boldsymbol{A}(\mathrm{m})$ of the tick data. To construct the long-term global recurrence plot, we modified the definition of recurrence plots as

$$
R_{i, j}(\boldsymbol{A}(m))= \begin{cases}1, & \text { if } d(\boldsymbol{A}(i), \boldsymbol{A}(j))<\min \left(r_{i}^{k}, r_{j}^{k}\right), \\ 0, & \text { otherwise, }\end{cases}
$$

where $r_{i}^{k}$ denotes the hamming distance between $A(i)$ and its $k$ th nearest neighbour. We used $k=8$.

We analysed MEG signals in a manner similar to the application for numerical simulations. The dimension of delay coordinates was $d=20$, and the length of segments was $S=20$. We set the fraction of points in both short-term local and longterm global recurrence plots as 0.1 .

1. Watts, D. J. \& Strogatz, S. H. Collective dynamics of 'small-world' networks. Nature 393, 440-442 (1998).

2. Lago-Fernández, L. F., Huerta, R., Corbacho, F. \& Sigüenza, J. A. Fast response and temporal coherent oscillations in small-world networks. Phys. Rev. Lett. 84, 2758 (2000).

3. Strogatz, S. H. Exploring complex networks. Nature 410, 268-276 (2001).

4. Schiff, S. J., So, P., Chang, T., Burke, R. E. \& Sauer, T. Detecting dynamical interdependence and generalized synchrony through mutual prediction in a neural ensemble. Phys. Rev. E 54, 6708-6724 (1996).

5. Arnhold, J., Grassberger, P., Lehnertz, K. \& Elger, C. E. A robust method for detecting interdependences: application to intracranially recorded EEG. Physica D 134, 419-430 (1999).

6. Chen, Y., Rangarajan, G., Feng, J. \& Ding, M. Analyzing multiple nonlinear time series with extended Granger causality. Phys. Lett. A 324, 26-35 (2004).

7. Yu, D., Righero, M. \& Kocarev, L. Estimating topology of networks. Phys. Rev. Lett. 97, 188701 (2006).

8. Napoletani, D. \& Sauer, T. Reconstructing the topology of sparsely connected dynamical networks. Phys. Rev. E 77, 026103 (2008).

9. Hirata, Y. \& Aihara, K. Identifying hidden common causes from bivariate time series: A method using recurrence plots. Phys. Rev. E 81, 016203 (2010).
10. Eckmann, J.-P., Kamphorst, S. O. \& Ruelle, D. Recurrence plots of dynamical Systems. Europhys. Lett. 4, 973-977 (1987).

11. Marwan, N., Romano, M. C., Thiel, M. \& Kurths, J. Recurrence plots for the analysis of complex systems. Phys. Rep. 438, 237-329 (2007).

12. Morris, M., Handcock, M. S. \& Hunter, D. R. Specification of exponential-family random graph models: terms and computational aspects. J. Stat. Softw. 24, 15487660 (2008).

13. Kantz, H. A robust method to estimate the maximal Lyapunov exponent of a time series. Phys. Lett. A 185, 77-87 (1994).

14. Tenenbaum, J. B., de Silva, V. \& Langford, J. C. A global geometric framework for nonlinear dimensionality reduction. Science 290, 2319-2323 (2000).

15. Romano, M. C., Thiel, M., Kurths, J., Kiss, I. Z. \& Hudson, J. L. Detection of synchronization for non-phase-coherent and non-stationary data. Europhys. Lett. 71, 466-472 (2005)

16. Neuhaus, W. Experimentelle Untersuchung der Scheinbewegung. Arch. Gesamte Psychol. 75, 315-458 (1930).

17. Ramachandran, V. S. \& Anstis, S. M. Perceptual organization in multistable apparent motion. Perception 14, 135-143 (1985).

18. Engel, A. K. \& Singer, W. Temporal binding and the neural correlates of sensory awareness. Trends Cogn. Sci. 5, 16-25 (2001).

19. Rodriguez, E. et al. The brainweb: phase synchronization and large-scale integration. Nature 397, 430-433 (1999).

20. Varela, F. J., Lachaux, J.-P., Rodriguez, E. \& Martinerie, J. Perception's shadow: long-distance synchronization of human brain activity. Nat. Rev. Neurosci. 2, 229-239 (2001).

21. Nakatani, H. \& van Leeuwen, C. Transient synchrony of distant brain areas and perceptual switching in ambiguous figures. Biol. Cybern. 94, 445-457 (2006).

22. Milo, R. et al. Network motifs: simple building blocks of complex networks. Science 298, 824-827 (2002).

23. de Vico Fallani, F. et al. Persistent patterns of interconnection in time-varying cortical networks estimated from high-resolution EEG recordings in humans during a simple motor act. J. Phys. A: Math. Theor. 41, 224014 (2008).

24. Bunke, H. \& Shearer, K. A graph distance metric based on the maximal common subgraph. Pattern Recognit. Lett. 19, 255-259 (1998).

25. Fernández, M.-L. \& Valiente, G. A graph distance metric combining maximum common subgraph and minimum common supergraph. Pattern Recognit. Lett. 22, 753-758 (2001)

26. Romano, M. C., Thiel, M., Kurths, J. \& von Bloh, W. Multivariate recurrence plots. Phys. Lett. A 330, 214 (2004).

27. Suzuki, S., Hirata, Y. \& Aihara, K. Definition of distance for marked point process data and its application to recurrence plot-based analysis of exchange tick data of foreign currencies. Int. J. Bifurcat. Chaos 20, 3699-3708 (2010).

28. Takens, F. Detecting strange attractors in turbulence. Springer Lect. Notes Math 898, 366-381 (1981).

\section{Acknowledgements}

We would like to thank Keita Araki for discussions. This research is partially supported by the Aihara Innovative Mathematical Modelling Project, the Japan Society for the Promotion of Science (JSPS) through the "Funding Program for World-Leading Innovative R\&D on Science and Technology (FIRST Program)," initiated by the Council for Science and Technology Policy (CSTP), and the Hokuriku Innovation Cluster for Health Science (MEXT Program for Fostering Regional Innovation).

\section{Author contributions}

$\mathrm{KI}, \mathrm{YH}, \mathrm{KA}$, and $\mathrm{HS}$ designed research, and $\mathrm{KI}$ and $\mathrm{YH}$ conducted the research and prepared the manuscript. KT and KW collected the MEG data. All the authors wrote and reviewed the manuscript.

\section{Additional information}

Supplementary information accompanies this paper at http://www.nature.com/ scientificreports

Competing financial interests: The authors declare no competing financial interests.

License: This work is licensed under a Creative Commons

Attribution-NonCommercial-ShareAlike 3.0 Unported License. To view a copy of this license, visit http://creativecommons.org/licenses/by-nc-sa/3.0/

How to cite this article: Iwayama, K. et al. Characterizing global evolutions of complex systems via intermediate network representations. Sci. Rep. 2, 423; DOI:10.1038/srep00423 (2012) 